CIC. Cuadernos de Información y Comunicación ISSN: 1135-7991

http://dx.doi.org/10.5209/ciyc.64637

\title{
Televisiones autonómicas y diversidad: análisis de la programación de Televisión del Principado de Asturias ${ }^{1}$
}

\author{
Azahara Cañedo ${ }^{2}$
}

Recibido: 1 de febrero de 2019/Aceptado: 25 de abril de 2019

Resumen. Este artículo, planteado desde la perspectiva investigadora de la Economía Política de la Comunicación y la Cultura, analiza la diversidad ofertada de $A 7$, la señal principal de Televisión del Principado de Asturias (TPA), desde su concepción de televisión pública de proximidad. La metodología aplicada se basa en una triangulación de datos que incluye un análisis documental, una propuesta propia de análisis de contenido de la parrilla de emisión, y la realización de entrevistas en profundidad a agentes clave implicados en el proceso de programación de TPA. Los resultados indican que $A 7$ ofrece un servicio público de proximidad que garantiza la dinamización del audiovisual regional ya que los contenidos asturianos son predominantes. En términos lingüísticos, a pesar de que la emisión en la lengua propia del territorio es minoritaria, los contenidos en asturiano han aumentado gradualmente su presencia y están íntegramente vinculados a la producción doméstica. Respecto a los macrogéneros, se programa una alta variedad de estos pero su presencia es desequilibrada: $A 7$ es una televisión informativa y de ficción que deja de lado el rol educativo de la televisión pública y la atención a minorías etarias.

Palabras clave: Televisión autonómica, diversidad audiovisual, servicio público de radiodifusión, programación televisiva, televisión del Principado de Asturias.

\section{[en] Community State television and diversity: analysis of the television programming of the Principality of Asturias}

\begin{abstract}
This article, posed from the research perspective of the Political Economy of Communication and Culture, analyses the diversity offered by A7, the main television channel of Televisión del Principado de Asturias (TPA), a specific case of public television of proximity. The methodology applied is based on a triangulation of data that includes a documentary analysis, an original proposal of content analysis, and various in-depth interviews with key agents involved in the TPA programming process. The results indicate that A7 offers a public service of proximity that guarantees the dynamisation of the regional audiovisual industry due to the fact that the Asturian contents are predominant. Concerning the diversity of language, despite the fact that the contents broadcasted in the regional language are minority, these are entirely linked to regional production and their presence has gradually increased. Regarding the genre, a high variety of genre is programmed in an unbalanced way: A7 is an informative and fiction television that leaves aside the educational role of public television and the attention to age group minorities.
\end{abstract}

1 Este artículo, que tiene como origen la tesis doctoral "Televisión del Principado de Asturias: dinamización y diversidad en el sector audiovisual asturiano (2005-2015)" (Cañedo, 2018), se enmarca en el proyecto de investigación "Diversidad audiovisual y plataformas en línea: el caso Netflix" (CSO2017-83539-R), financiado por la Agencia Estatal de Investigación (AEI), en el marco del Programa Estatal de I+D+i Orientada a los Retos de la Sociedad del Ministerio de Ciencia, Innovación y Universidades de España, y por el Fondo Europeo de Desarrollo Regional (FEDER) de la Unión Europea.

2 Universidad Carlos III de Madrid. 
Keywords: Regional television, Audiovisual Diversity, Public Service Broadcasting, TV Programming, Television del Principado de Asturias.

Sumario. 1. Introducción. 1.1. Televisiones autonómicas y diversidad. 1.2. Medir la diversidad en televisión. 1.3. Televisión del Principado de Asturias. 1.4. Preguntas de investigación. 2. Metodología. 3. Discusión de resultados. 3.1. Diversidad en el origen geográfico. 3.2. Diversidad en la lengua de emisión. 3.3. Diversidad en los macrogéneros. 4. Conclusiones y propuestas. 5. Referencias bibliográficas.

Cómo citar: Cañedo, A. (2019). Televisiones autonómicas y diversidad: análisis de la programación de Televisión del Principado de Asturias, en CIC. Cuadernos de Información y Comunicación 24, 113-133.

\section{Introducción}

En un mundo globalizado y regido por leyes fundamentalmente económicas, la falta de protección de las expresiones culturales minoritarias se pone de manifiesto en el creciente interés que ha adquirido, desde perspectivas de análisis crítico, la cuestión de la diversidad cultural (Mattelart, 2006; Albornoz y García Leiva, 2017a y 2017b). El reconocimiento de la diversidad es hoy "una definición de salud social" (Zallo en Albornoz, 2011:33) y se ha convertido en protagonista de las políticas de comunicación. Estas, focalizadas en la dimensión cultural del desarrollo, buscan contrarrestar las debilidades de un sistema audiovisual caracterizado por una "saturación de los mercados que impide la visibilidad de los productos innovadores o minoritarios, de las óperas primas, de las pequeñas y medianas empresas y de las lenguas y culturas minoritarias" (Bustamante en Albornoz y García Leiva, 2017b: 19).

Desde la perspectiva investigadora de la Economía Política de la Comunicación y la Cultura, el debate sobre la diversidad va ligado al del servicio público. La televisión pública es indispensable "para cumplir las funciones de refuerzo de las identidades, la defensa de las lenguas, la promoción de la cohesión social, el fomento de la participación democrática y la creación de consenso" (Prado en Marzal et al., 2015: 59). Así, aunque el servicio público se encuentra en constante redefinición en el área de la comunicación (Richeri, 1983; Pasquali, 1991; Murdock, 1994; UNESCO, 2006), es concebido como instrumento de democratización y reflejo de la identidad cultural de los territorios.

En Europa, donde el servicio público de radiodifusión es "una seña de identidad" (Bustamante y García Leiva en Gutiérrez, 2017: 21), la televisión pública se define como garante de una programación plural y de producción propia doméstica, protectora de las minorías, alfabetizadora, y regida por los principios del pluralismo interno y la objetividad. Así, se ha constatado que la oferta pública presenta mayor diversidad que la de la televisión privada (Van der Wurff, 2004 y 2005; Prado y Delgado 2010; Prado en Marzal et al., 2015; Prado en Albornoz y García Leiva, 2017a; Delgado et al., 2017).

\subsection{Televisiones autonómicas y diversidad}

El debate sobre la diversidad ha ido en paralelo a la emergencia, como sujeto activo, de los ámbitos territoriales inferiores al Estado-nación, fenómeno que ha desembocado en la territorialización de la investigación en cultura y comunicación. Si los productos comunicacionales son valores de identidad, la diversidad es la herramienta para "que 
una nación se conciba a sí misma y para garantizar su cohesión social" (Frau-Meigs, 2002: 8). El reconocimiento del rol de la televisión pública en ello es clave.

El binomio televisión pública-televisión regional cobra un papel fundamental ya que "la televisión de proximidad es, por su propia naturaleza, la de la diversidad" (Moragas et al., 1999: 22). La vinculación entre comunicación de proximidad y diversidad se refleja en la Convención sobre la Protección y Promoción de la Diversidad de las Expresiones Culturales (UNESCO, 2005), primer instrumento jurídico específico sobre diversidad cultural en el que, además, se reconoce a la diversidad lingüística como un elemento fundamental de la misma.

En España, las televisiones autonómicas son las encargadas de reflejar la diversidad de los territorios en que operan, y así se señala en sus leyes de creación y normativas rectoras. A pesar de las diferencias encontradas en función del contexto histórico, político y económico en el que han nacido cada una de estas televisiones, su legitimidad está justificada, entre otros objetivos, en la promoción de la diversidad cultural y lingüística (Albornoz y Cañedo en Marzal, 2015; Campos Freire, 2016). Cumplir con el mandato de servicio público encomendado se vincula a la obligación de afrontar las demandas específicas de sus autonomías. Esto pasa por impulsar la producción propia de programas, garantizar una programación diversa y de calidad en la que se priorice la información regional, impulsar la normalización lingüística y articular un espacio comunicativo propio (Zallo, 2011: 332-333).

La mayor parte de los trabajos académicos sobre televisiones autonómicas se han centrado en estudios de caso sobre la relación diversidad-servicio público referida a los contenidos. En aquellas televisiones autonómicas en las que se ha fijado la labor de servicio público en mandatos marco y contratos programa ${ }^{3}$, se han establecido cuotas de programación al respecto de tres indicadores: el origen de la producción de los programas, la clasificación por géneros de los mismos y el idioma de emisión.

En primer lugar, Promover la diversidad lingüística es fundamental en un estado en el que el idioma oficial es el castellano, pero se hablan otras lenguas, algunas reconocidas cooficialmente (catalán, valenciano, gallego, euskera y occitano aranés) y otras con diferentes grados de reconocimiento jurídico (asturiano, aragonés y fala). Por su parte, la diversidad sobre el origen de los contenidos está relacionada con el objetivo de estas televisiones de crear sectores audiovisuales sólidos en los territorios en los que operan. Por último, la diversidad genérica de los programas se relaciona con la satisfacción de los diferentes gustos y preferencias de la sociedad y la influencia de los géneros en las relaciones productivas que se establecen en el mercado de las industrias culturales. En este sentido, siempre y cuando los contenidos cumplan con los requisitos de calidad, responsabilidad social, desarrollo cultural e

\footnotetext{
En el marco de la legislación europea se reconocen dos instrumentos para concretar los objetivos de servicio público: el mandato marco y el contrato programa. El mandato marco es el documento en el que se concretan los objetivos generales del servicio público esencial de radiodifusión para un determinado operador público. En España, la Ley General de la Comunicación Audiovisual (2010) señala que los objetivos de servicio público deberán concretarse cada nueve años por los parlamentos u órganos similares a nivel autonómico y local. Por su parte, el contrato programa es el documento donde se especifican los compromisos establecidos en el mandato marco y en el que se incluye el sistema de fiscalización a lo largo de un plazo de tiempo determinado (su duración suele variar entre los dos o tres años). Los operadores autonómicos que, con fecha de mayo de 2019, tienen vigentes sus contratos programa son Euskal Irrati Telebista, Corporación Aragonesa de Radio y Televisión, Agencia Pública Empresarial de la Radio Televisión de Andalucía, Corporació Valenciana de Mitjans de Comunicació, Radiotelevisión de la Región de Murcia y Radiotelevisión Madrid.
} 
independencia informativa; todos los géneros deben estar presentes en las parrillas de emisión de las televisiones públicas (Prado en Marzal et al., 2015: 57).

\subsection{Medir la diversidad en televisión}

Medir la diversidad en el audiovisual es una de las principales problemáticas surgidas en torno al concepto y son varios los trabajos que reflexionan al respecto. Dennis McQuail (1998) es pionero en identificar puntos estructurales para el estudio de la diversidad y aporta al corpus teórico una de las principales dicotomías sobre el concepto: diversidad externa - propia de un sistema de medios en su conjunto- y diversidad interna -propia de un medio de comunicación concreto-. En ambas dimensiones, la diversidad es, para McQuail, un reflejo de la sociedad.

Por su parte, Philip Napoli (1999) descompone el concepto en tres componentes: diversidad de las fuentes de producción, diversidad de los contenidos y diversidad de exposición, entendida ésta como el consumo de contenidos simbólicos por parte de los receptores. Napoli reclama que se preste atención no solo la diversidad emitida sino también la recibida, que ha estado tradicionalmente ausente de los análisis, porque entiende que la emisión de una parrilla de programación diversa no es directamente proporcional a un consumo diverso por parte de la audiencia.

El trabajo de Andrew Stirling (1998 y 2007), aunque no pertenece al campo de los estudios de comunicación, es referencia en el análisis de la diversidad porque propone tres propiedades para medirla: variedad, balance y disparidad. Tres factores que, aunque independientes, la supresión de uno de ellos del paradigma cuestionaría el resultado de la medición. La principal crítica a Stirling desde la teoría comunicativa radica en la dificultad de aplicar el factor de la disparidad a los productos mediáticos, ya que medir la diferencia entre las diferentes expresiones culturales arroja múltiples dudas (Bustamante en Albornoz y García Leiva, 2017a: 150).

Diversas investigaciones (Ranaivoson, 2005 y 2007; UNESCO-UIS, 2011), sin embargo, certifican la validez de la propuesta de Stirling, pero confirman la necesidad de nuevos ajustes en su acercamiento metodológico. Uno de los principales problemas es la ausencia de una definición válida del concepto de diversidad en el audiovisual. Ante esto, el grupo de investigación Diversidad Audiovisual ${ }^{4}$, a partir de los trabajos previos mencionados, plantea que la diversidad en el audiovisual depende de una multiplicidad de factores. Para su evaluación ha de tenerse en cuenta que:

- La capacidad de producción, distribución y exhibición/emisión de contenidos audiovisuales no esté concentrada en un número reducido de agentes y que estos agentes sean de diferentes tipos de titularidad, tamaño y origen geográfico.

- Los contenidos audiovisuales exhiban diferencias de variedad, balance y disparidad en términos de valores, identidad y estética. Estos deben reflejar a los múltiples grupos que conviven en una determinada sociedad y hacerse eco de culturas foráneas.

- Los ciudadanos puedan acceder y elegir entre un elevado número de contenidos audiovisuales e, incluso, puedan crearlos y difundirlos. (Albornoz y García Leiva, 2017b: 32)

Más información en: www.diversidadaudiovisual.org 
Así, se propone un modelo de medición de la diversidad en televisión que afecta a diferentes dimensiones del proceso televisivo, no excluyentes entre sí, lo que implica la posibilidad de cuantificar la diversidad en función de múltiples factores (gráfico 1). A partir del trabajo de Napoli y Stirling, la diversidad se divide en tres áreas de aplicación (fuentes, contenidos y visionado), descompuestas en diferentes subcomponentes que son analizados en términos de variedad, equilibrio y disparidad.

Gráfico 1. Modelo de medición de la diversidad en televisión propuesto por Marenghi.

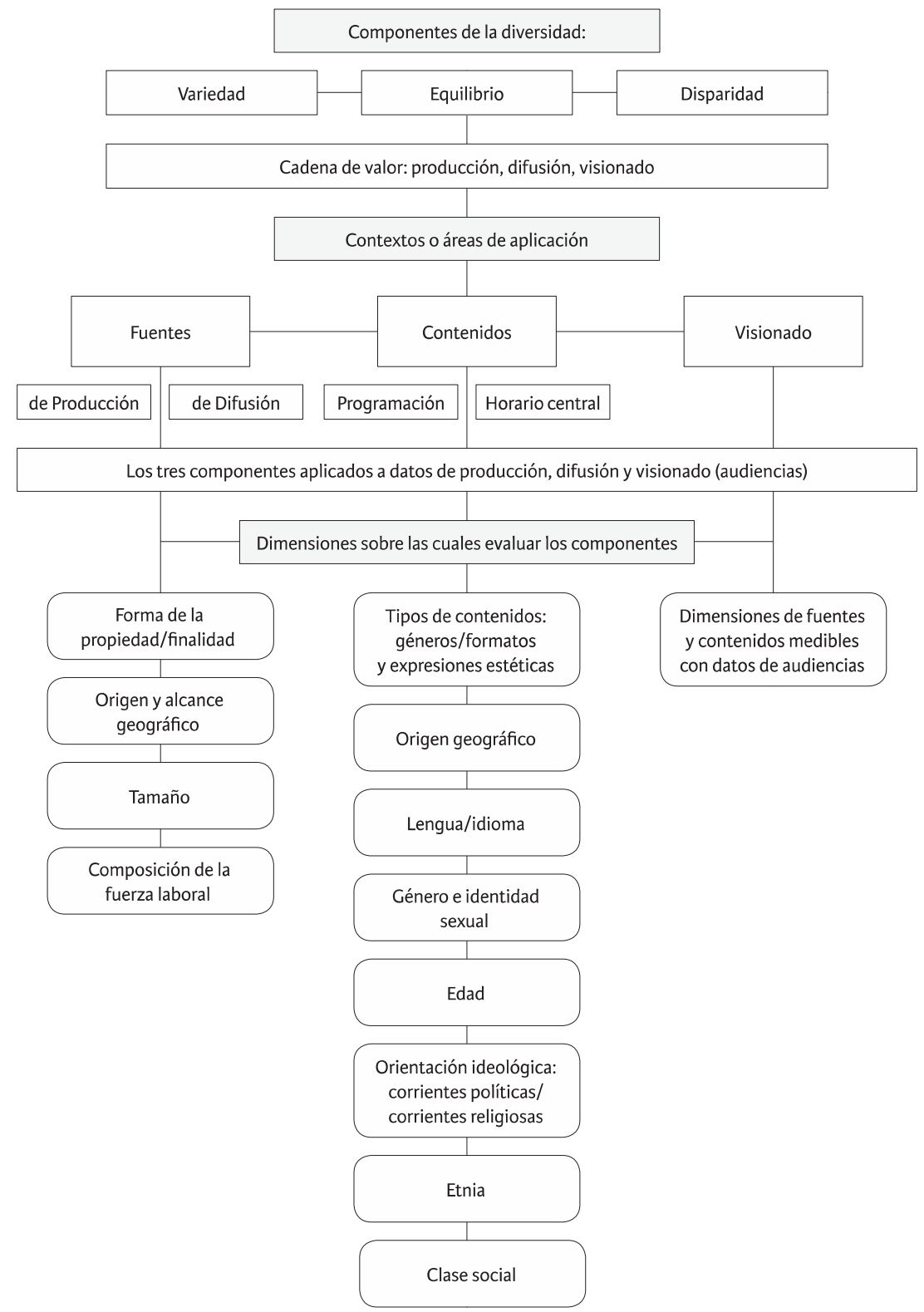

Fuente: Marenghi en Albornoz y García Leiva, 2017b: 227. 


\subsection{Televisión del Principado de Asturias}

Televisión del Principado de Asturias, objeto de estudio de este trabajo, es la televisión autonómica del Principado de Asturias -o, simplemente, Asturias-, una comunidad autónoma española uniprovincial, con una superficie del $2 \%$ del territorio estatal y poco más de un millón de habitantes. La región es la $13^{\mathrm{a}}$ economía española por volumen de PIB, y presenta un PIB per cápita (22.046€ en 2017) y una tasa de paro (14,6\% en 2017) ligeramente inferiores a la media española, si bien el desempleo afecta principalmente a los jóvenes. Esto, unido a la alta mortalidad y la baja natalidad hace que, desde el inicio de la crisis económica de 2008, la región se encuentre en constante decrecimiento poblacional. A nivel empresarial, Asturias es principalmente una región industrial. El volumen de su sector audiovisual, altamente atomizado y precarizado, representa apenas el 1,3\% del sector español (DIRCE, 2017).

En términos identitarios, Asturias destaca por su diversidad lingüística ya que en la región cohabitan el castellano y el asturiano, la lengua propia del territorio, reconocida en el Estatuto de Autonomía para Asturias (1981), pero sin rango de cooficialidad y en situación de minorización lingüística. A pesar de que en 1998 se aprobó una ley sobre su uso y promoción ${ }^{5}$, esta no establece obligaciones sino que marca solo algunas recomendaciones. Desde 2001, el asturiano está incluido en el Atlas of the World's languages in danger of dissaperaing (Atlas mundial de lenguas en peligro de desaparición) de la UNESCO. En septiembre de 2017, la Federación Socialista Asturiana (FSA-PSOE), primera fuerza política del momento, acordó plantear la cooficialidad de la lengua en una futura reforma del Estatuto de Autonomía. Este posicionamiento, en sintonía con las reivindicaciones históricos de otros partidos de ideología progresista con menor peso político en la región, posicionó el debate en las instituciones y en la sociedad. Estudios recientes señalan que el $86,1 \%$ de los jóvenes asturianos está a favor de la cooficialidad (Hevia, 2018: 18).

Respecto a los medios de comunicación públicos regionales, su desarrollo en Asturias se considera tardío con respecto otras autonomías ya que, hasta la reforma de 1999, el Estatuto de Autonomía no incluía en su redacción la competencia constitucional para regular, crear y mantener una televisión pública propia. Cuatro años después, la ley del Principado de Asturias 2/2003, de 17 de marzo, de Medios de Comunicación Social fue la encargada de crear el Ente Público de Comunicación de Principado de Asturias (EPCPA), compuesto por tres sociedades gestoras ${ }^{6}$.

En su trayectoria, el EPCPA ha tenido tres directores generales que han marcado distintos rumbos en su historia: José Ramón Pérez Ornia (2005-2011), Juan José Guerenabarrena (2011) y Antonio Virgili (2012-2015). A lo largo de este período, la televisión autonómica ha puesto en emisión tres señales televisivas diferencias: $A 7^{7}$, la señal principal, que emite regularmente desde el 9 de junio de 2006; $A 8$, el segundo canal, que emite por primera vez el 26 de mayo de 2007; y $A 9 H D$, destinada a la emisión en simulcast en alta definición, en emisión desde el 27 de marzo de 2010.

\footnotetext{
Ley del Principado de Asturias 1/1998, de 23 de marzo, de uso y promoción del bable/asturiano.

Las tres sociedades gestoras del EPCPA eran Televisión del Principado de Asturias S.A.U.; Radio del Principado de Asturias S.A.U., y Productora de Programas del Principado de Asturias S.A. (PRODA).

Las señales $A 7$ y $A 8$ operan bajo esta denominación desde el 21 de diciembre de 2009. Anteriormente eran designadas TPA1 y TPA2, respectivamente. Para facilitar la comprensión del texto, se ha optado por usar la denominación actual de ambas señales.
} 
Mantener programaciones independientes para las dos señales principales no ha sido posible por los bajos presupuestos de actividad de TPA: en el período 2006-2015, se han mantenido como los más bajos de todas las corporaciones agrupadas en la Federación de organismos de radio y televisión autonómicos (FORTA), de la que el EPCPA forma parte.

La concepción de la radiotelevisión autonómica asturiana como reflejo cultural es inherente a su ley de creación que señala que el EPCPA nace para "potenciar las peculiaridades y el afianzamiento de la identidad asturiana, a través de la difusión, conocimiento y desarrollo de los valores históricos y culturales en toda su variedad y riqueza" (Asturias, Ley 2/2003). Aunque esta ley no contempla en su articulado el desarrollo de mecanismos que pueden establecer cuotas y fijar compromisos de programación para el cumplimiento del servicio público, como el mandato marco o el contrato programa; al inicio de las emisiones regulares de TPA, el Consejo de Administración aprueba los principios y criterios de programación de los medios del ente público de comunicación del Principado de Asturias (EPCPA, 2006). Este documento funciona a modo de guía rectora del modelo programático de la radiotelevisión pública que se concibe sobre tres pilares clave: servicio público, diversidad lingüística y dinamización del sector audiovisual asturiano, caracterizado tradicionalmente por su alta atomización, precariedad y polarización (Cañedo, 2018: 205). En él se señalan, como objetivos prioritarios: impulsar la producción audiovisual independiente asturiana y garantizar la presencia en la parrilla de producciones audiovisuales comunitarias y latinoamericanas; ofertar una programación de servicio público diversa en cuanto a los contenidos; y promocionar el asturiano como lengua propia. Si bien, a este último respecto, la ya mencionada no cooficialidad de la lengua y la ausencia de una política lingüística sobre medios de comunicación, dificulta el cumplimiento de un objetivo compartido con otras televisiones autonómicas que operan en territorios con lengua propia.

En 2014, la Junta General del Principado de Asturias aprobó la Ley 8/2014, de 17 de julio, de segunda reestructuración del sector público autonómico. La ley, elimina el EPCPA y crea una única sociedad gestora, Radiotelevisión del Principado de Asturias (RTPA), por absorción de las tres anteriores. Además, de acuerdo a lo estipulado en la Ley General de la Comunicación Audiovisual (2010), establece la obligación de aprobar un mandato marco y sucesivos contratos programa para el correcto desarrollo del servicio público de radiotelevisión. Sin embargo, aunque RTPA comenzó su actividad en enero de 2015, hasta la fecha, estos mecanismos no han sido desarrollados.

\subsection{Preguntas de investigación}

Este artículo se pregunta sobre la naturaleza de los contenidos que ha emitido TPA, en su primera década de funcionamiento (2005-2015). El análisis se centra en la diversidad de contenidos ofertada por esta televisión autonómica (diversidad interna) a la que se le presupone una programación de servicio público de proximidad enfocada a Asturias.

De acuerdo a lo señalado en el documento que recoge los principios y criterios de programación del EPCPA y a partir del modelo propuesto por el grupo de investigación Diversidad Audiovisual para la medición de la diversidad en los sistemas televisivos, se plantean varias preguntas de investigación. Estas preguntas están relacio- 
nadas con la diversidad de contenidos emitidos en términos de origen geográfico de la producción, la lengua de emisión y los macrogéneros.

La primera pregunta que se plantea es: ¿cuál es el origen de los contenidos emitidos por TPA y cuál es su proporción de presencia en función de los diferentes macrogéneros? En segundo lugar, respecto a la lengua de emisión y con la intención de discriminar positivamente la presencia del asturiano en la parrilla de programación de $A 7$, se pregunta: ¿cuánto contenido se ha emitido en asturiano y cuál ha sido la evolución de su presencia en el período de análisis? Una vez definido esto: ¿quién ha producido los contenidos en lengua asturiana, en qué macrogéneros predomina su presencia y en qué horario se emiten? Por último, respecto a los macrogéneros, la pregunta que se plantea es: ¿cuál ha sido el peso de presencia de los diferentes macrogéneros en la parrilla de programación de $A 7$ ?

\section{Metodología}

La metodología aplicada para responder a las preguntas de investigación planteadas se basa en una triangulación de datos que incluye diferentes métodos científicos. En primer lugar, se ha realizado un análisis documental de fuentes de diversa índole (bibliografía, hemeroteca de prensa, informes sectoriales, legislación y documentación interna del EPCPA y de TPA). En segundo lugar, dada la escasa producción documental al respecto de nuestro objeto de estudio y las lagunas de información localizadas durante la investigación, se han desarrollado entrevistas en profundidad a agentes clave implicados en el proceso de programación de TPA. En este sentido, se destacan por su especial valor informativo las mantenidas con los tres directores generales que ha tenido el EPCPA durante el período analizado: José Ramón Pérez Ornia, Juan José Guerenabarrena y Antonio Virgili. Finalmente, se ha ejecutado un análisis de contenido de la parrilla de emisión del canal principal de TPA, $A 7$, en el período comprendido entre el inicio de las emisiones regulares (junio de 2006) y su reestructuración empresarial (enero de 2015).

El análisis de contenido realizado responde a una propuesta metodológica propia. La muestra, que se ha adaptado al contexto sociopolítico y empresarial de TPA y a las características de la parrilla de programación a analizar, abarca el análisis de dos semanas completas (lunes a domingo sin feriados) por año, durante los meses de marzo y noviembre entre el segundo semestre de 2006 y el año 2014. Esto computa un total de 119 días analizados. Se ha considerado como unidad de análisis cada uno de los programas que integran las parrillas de programación de $A 7$ en las semanas seleccionadas. En total, se han analizado 2.439 programas (130.659 minutos de emisión), que se han sometido al análisis de las once variables que componen el protocolo de análisis: título del contenido, empresa productora, fecha de emisión, horario de emisión, día de la semana, franja horaria8, ciclo de la semana, duración

\footnotetext{
A la hora de definir las franjas horarias, una primera opción metodológica por la que se optó fue por trabajar con la clasificación propuesta por el observatorio Euromonitor (Prado y Delgado, 2010) que plantea el reparto de seis franjas horarias entre las 07:00 y las 01:00 horas del día. Sin embargo, dado que TPA estructura la parrilla de programación de $A 7$ en una clasificación propia de ocho franjas horarias repartidas entre las 24 horas del día; se ha definido una propuesta propia de franjas. Se propone una clasificación en seis franjas horarias que se extiende entre las 7:00 y las 02:30 horas, debido a que no en todos los períodos de emisión de TPA la programación ha completado el ciclo diario y, aún cuando lo ha hecho, ha utilizado las madrugadas para la emisión de reposiciones o contenidos de webcam.
} 
del programa (en minutos), origen de la producción, lengua de emisión y macrogénero.

Para el análisis específico de la diversidad, de acuerdo a las preguntas de investigación planteadas, se establecen tres variables centrales del análisis. La primera tiene que ver con el origen de la producción de los contenidos emitidos, una de las posibles caras "del prisma desde el que se puede medir la diversidad de las programaciones televisiva, más allá del género" (Prado en Albornoz y García Leiva, 2017a: 180). Esta dimensión es determinante si se tiene en cuenta el objetivo de servicio público de dinamizar el sector audiovisual asturiano. La segunda variable tiene que ver con la lengua/idioma de emisión de los contenidos televisivos emitidos. La diversidad lingüística es una de las razones que justifica la existencia de TPA al ser el Principado de Asturias una región con lengua propia que posee mecanismos legales de protección y promoción. La tercera variable se refiere al género de los contenidos emitidos, otra de las posibles dimensiones para medir la diversidad y la más común por la facilidad de su cuantificación y análisis (Van der Wurff, 2005: 250). A este respecto, se utiliza la clasificación definida por el observatorio Euromonitor, la cual se estructura en tres niveles de clasificación (macrogénero, género y microgénero), considerándose para este artículo únicamente el primer nivel de análisis.

Tras definir el protocolo, se prepararon las fichas de análisis y se procedió al volcado de información. Al respecto, se señalan las siguientes decisiones metodológicas, tomadas a raíz de la naturaleza de la muestra y que no modifican la relevancia ni la validez de los resultados ya que su incidencia en los mismos es mínima:

- En lo que respecta a la duración de los contenidos, el minutaje se ajusta al horario programado de emisión del contenido sin incluir espacios publicitarios.

- Las parrillas de programación no incluyen avances informativos ni microcápsulas de contenido inferiores a cinco minutos de duración, por tanto, estos también se excluyen del cómputo.

Finalmente, la diversidad presente en los contenidos emitidos es analizada en términos cuantitativos de tiempo de emisión y evolución de su presencia en el flujo de programación, aplicando los factores de variedad y equilibrio indicados por Stirling. Una vez obtenidos los datos cuantitativos, estos se valoran en términos cualitativos tras la aplicación del análisis de contingencias que pone en valor los resultados obtenidos tras el cruce de datos. 
Tabla 1. Propuesta metodológica de análisis de contenido: muestra y variables de análisis.

\begin{tabular}{|c|c|}
\hline \multicolumn{2}{|r|}{ Muestra } \\
\hline Año & Semanas \\
\hline 2006 & 13 a 19 de noviembre \\
\hline 2007 & $\begin{array}{l}12 \text { a } 18 \text { de marzo } \\
12 \text { a } 18 \text { de noviembre }\end{array}$ \\
\hline 2008 & $\begin{array}{l}10 \text { a } 16 \text { de marzo } \\
10 \text { a } 16 \text { de noviembre }\end{array}$ \\
\hline 2009 & $\begin{array}{l}9 \text { a } 15 \text { de marzo } \\
9 \text { a } 15 \text { de noviembre }\end{array}$ \\
\hline 2010 & $\begin{array}{l}15 \text { a } 21 \text { de marzo } \\
15 \text { a } 21 \text { de noviembre }\end{array}$ \\
\hline 2011 & $\begin{array}{l}14 \text { a } 20 \text { de marzo } \\
14 \text { a } 20 \text { de noviembre }\end{array}$ \\
\hline 2012 & $\begin{array}{l}12 \text { a } 18 \text { de marzo } \\
12 \text { a } 18 \text { de noviembre }\end{array}$ \\
\hline 2013 & $\begin{array}{l}11 \text { a } 17 \text { de marzo } \\
11 \text { a } 17 \text { de noviembre }\end{array}$ \\
\hline 2014 & $\begin{array}{l}10 \text { a } 16 \text { de marzo } \\
10 \text { a } 16 \text { de noviembre }\end{array}$ \\
\hline
\end{tabular}

\begin{tabular}{|c|c|}
\hline \multicolumn{2}{|c|}{ Variables de análisis } \\
\hline Variable & Elementos \\
\hline Título del contenido & \\
\hline Empresa productora & \\
\hline Fecha de emisión & \\
\hline Día de emisión & $\begin{array}{l}\text { Lunes } \\
\text { Martes } \\
\text { Miércoles } \\
\text { Jueves } \\
\text { Viernes } \\
\text { Sábado } \\
\text { Domingo }\end{array}$ \\
\hline Horario de emisión & \\
\hline Franja horaria & $\begin{array}{l}\text { Mañana }(7: 00-13: 30 \mathrm{~h}) \\
\text { Mediodía }(13: 30-15: 00 \mathrm{~h}) \\
\text { Sobremesa }(15: 00-18: 00 \mathrm{~h}) \\
\text { Tarde }(18: 00-20: 30 \mathrm{~h}) \\
\text { Prime-Time }(20: 30-0: 30 \mathrm{~h}) \\
\text { Noche }(0: 30-2: 30 \mathrm{~h})\end{array}$ \\
\hline Ciclo de la semana & $\begin{array}{l}\text { Laborable } \\
\text { Fin de semana }\end{array}$ \\
\hline Duración (en minutos & \\
\hline Lengua de emisión & $\begin{array}{l}\text { Asturiano } \\
\text { Castellano } \\
\text { Otra }\end{array}$ \\
\hline Macrogénero & $\begin{array}{l}\text { Ficción } \\
\text { Información } \\
\text { Info-Show } \\
\text { Show } \\
\text { Concurso } \\
\text { Deportes } \\
\text { Infantil } \\
\text { Juvenil } \\
\text { Educación } \\
\text { Religión } \\
\text { Diversos }\end{array}$ \\
\hline
\end{tabular}


Tabla 2. Propuesta metodológica de análisis de contenido: cómputo de resultados (análisis de contingencias).

\begin{tabular}{|l|l|l|}
\hline \multicolumn{1}{|c|}{ Variable 1 } & \multicolumn{1}{|c|}{ Variable 2 } & \multicolumn{1}{c|}{ Valoración cualitativa } \\
\hline Origen geográfico & Lengua & $\begin{array}{l}\text { ¿Qué relaciones se establecen entre el origen geográfico de la } \\
\text { producción y la lengua de emisión? }\end{array}$ \\
\hline Origen geográfico & Macrogénero & $\begin{array}{l}\text { ¿Qué relaciones se establecen entre el origen geográfico } \\
\text { de la producción y el macrogénero en el que se inserta el } \\
\text { contenido? }\end{array}$ \\
\hline Lengua & Macrogénero & $\begin{array}{l}\text { ¿Qué relaciones se establecen entre la lengua de emisión y el } \\
\text { macrogénero en el que se inserta el contenido? }\end{array}$ \\
\hline
\end{tabular}

Fuente: Elaboración propia.

\section{Discusión de resultados}

\subsection{Diversidad en el origen geográfico}

El análisis de la diversidad en el origen geográfico de los contenidos emitidos ratifica que $A 7$ cumple con el propósito de dinamizar el sector audiovisual asturiano ya que el $62,8 \%$ del tiempo de emisión tiene su origen en Asturias. Como señala José Ramón Pérez Ornia (entrevista personal, 2018), desde un principio "intentamos que Asturias tuviera una presencia muy fuerte en la televisión autonómica".

Sin embargo, la máxima cifra de presencia de los contenidos de origen asturiano se detecta en el bienio 2013-2014, bajo la dirección general de Antonio Virgili. Los dos últimos años de actividad de TPA, los contenidos originados en Asturias alcanzan el $71 \%$ del tiempo de emisión, siguiendo con la tendencia de las televisiones públicas europeas que se caracterizan por una mayor presencia de contenidos domésticos (Delgado et al., 2017: 139). En este período se constata, además, una mayor variedad de empresas asturianas contratadas.

La alta presencia de contenidos originados en Asturias en la emisión de la señal principal de TPA evidencia el aporte a la diversidad en el audiovisual de esta televisión autonómica. Durante todo el período analizado, los contenidos asturianos superan el 50\% del tiempo de emisión anual y están presentes en todas las franjas horarias lo que, en un contexto en el que no se han establecido cuotas de obligado cumplimiento ante la ausencia de mandato marco y contratos programa, evidencia la apuesta por el cumplimiento efectivo del servicio público.

El segundo origen con mayor presencia en la parrilla de emisión de $A 7$ es Estados Unidos (19,1\%). En los bienios 2008-2009 y 2011-2012, los contenidos estadounidenses superan el umbral del $20 \%$ y alcanzan una media de presencia en torno al $25 \%$. Este dato supone la reafirmación de la hegemonía de este país en el mercado audiovisual mundial, especialmente como productores de ficción (Delgado et al., 2017: 136). En $A 7$, el 96\% de los contenidos estadounidenses son del macrogénero Ficción, quedando reducida a mínimos la presencia de otros macrogéneros: un 2\% de Información (documentales de National Geographic); y un 2\% del macrogénero Deportes (retransmisión del mundial de automovilismo de Fórmula 1). 
Gráfico 2. A7-TPA: Origen geográfico de los programas emitidos, 2006-2014

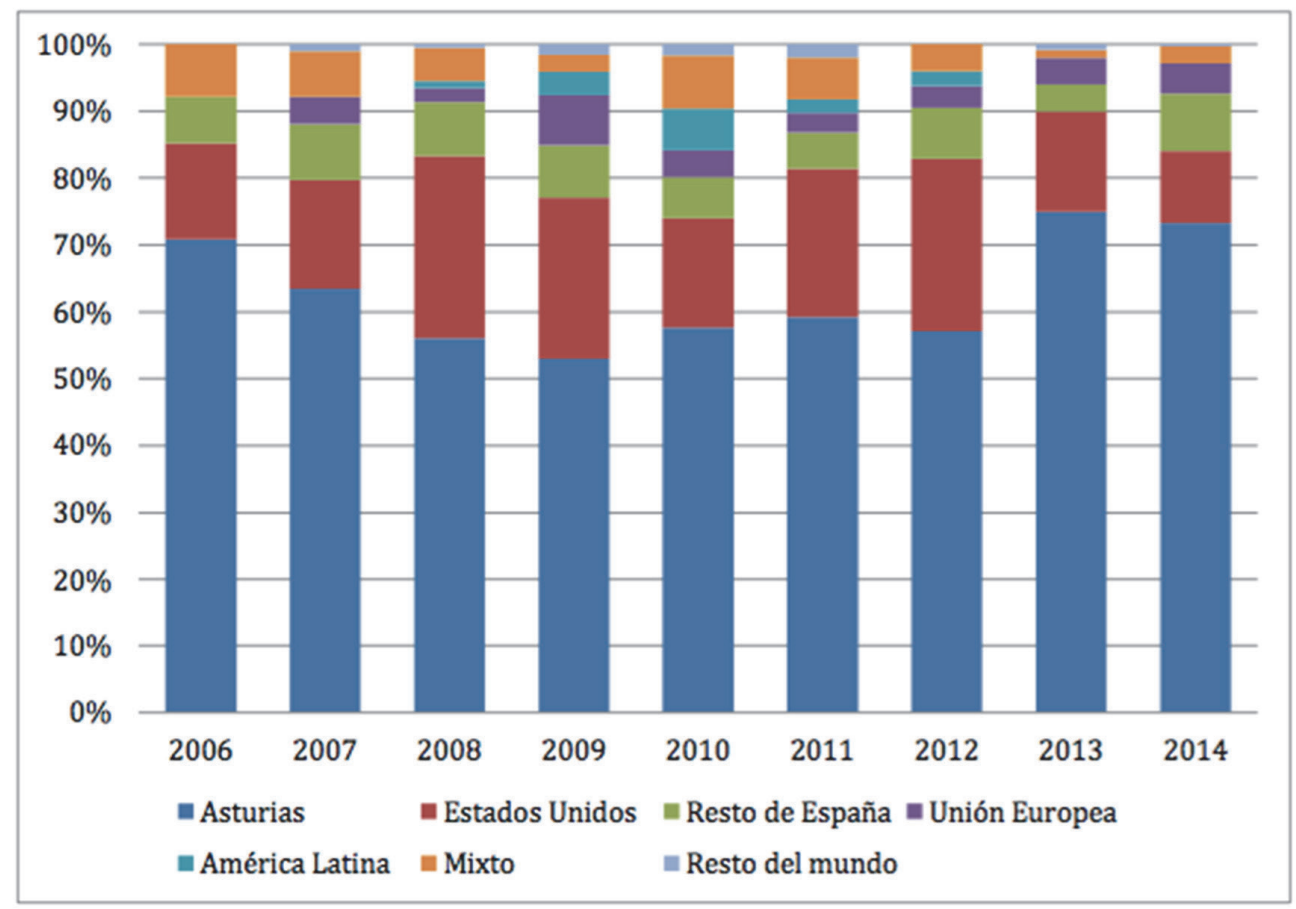

Fuente: Elaboración propia.

El análisis de los resultados permite, además, identificar cierta reciprocidad entre los incrementos/decrementos de la presencia de contenidos originados en Estados Unidos con respecto a los programas de origen asturiano. Por regla general, cuanto más alta es la presencia de estos últimos, más disminuye la de los programas estadounidenses. Esto indica que la pauta de construcción de la parrilla de programación se articula en torno a la preeminencia de contenidos provenientes de estos dos orígenes.

Los contenidos originados en el resto de la Unión Europea o en América Latina, con los que los principios y criterios de programación del EPCPA habían fijado un compromiso de promoción, apenas representan un 5,3\% del total del tiempo de emisión. Además, la presencia de estos contenidos en la parrilla de programación es inestable y si se analizan los países de origen apenas existe variedad entre ellos. En el caso del resto de la Unión Europea, se localizan producciones de siete países (Reino Unido, Rumanía, Francia, Alemania, Yugoslavia, Italia y Austria) pertenecientes a tan solo tres de los once macrogéneros de análisis. Estos son: Ficción, Información y Deportes; los dos últimos con una presencia discontinua durante el período analizado. Por su parte, los contenidos catalogados como de origen América Latina son todo telenovelas que provienen de dos países, México y Venezuela, los cuales poseen una posición hegemónica en el mercado latinoamericano de la producción de ficción (Mazzioti, 1996).

La categoría Resto del mundo, supone la menor presencia, con una media inferior al 1\% del tiempo de emisión, y no sigue ningún patrón de actuación definido, 
con períodos de ausencia. Los contenidos de este origen no presentan variedad ya que son solo contenidos de Ficción $(75,4 \%)$ y Show $(24,5 \%)$ producidos por Canadá y Australia. En este sentido, aunque dentro del contenedor infantil "Presta asgaya" se emiten algunas series de animación producidas en Japón, estas se categorizan como de origen mixto dado que la edición del programa es de origen asturiano.

Estos contenidos de origen mixto ocupan una media anual del 4,9\% del tiempo de presencia pero no existe patrón de regularidad definido al respecto, ni en los orígenes ni en los macrogéneros. Tampoco presentan patrón de estabilidad los contenidos producidos en el resto de España. Aunque estos se emiten durante todo el período analizado, con una media anual de presencia del $7 \%$, se detecta un alto grado de renovación de los programas.

Si se pone en relación el origen de las producciones con los macrogéneros que representan, es determinante señalar que $A 7$ cumple efectivamente con el servicio público de proximidad ya que emite una televisión informativa preeminentemente asturiana. Del total de contenidos informativos emitidos, el $91 \%$ se producen en la región, representando este dato el $65,3 \%$ del volumen total de contenidos originados en Asturias.

Gráfico 3. A7-TPA: Contenidos de información emitidos según origen, 2006-2014

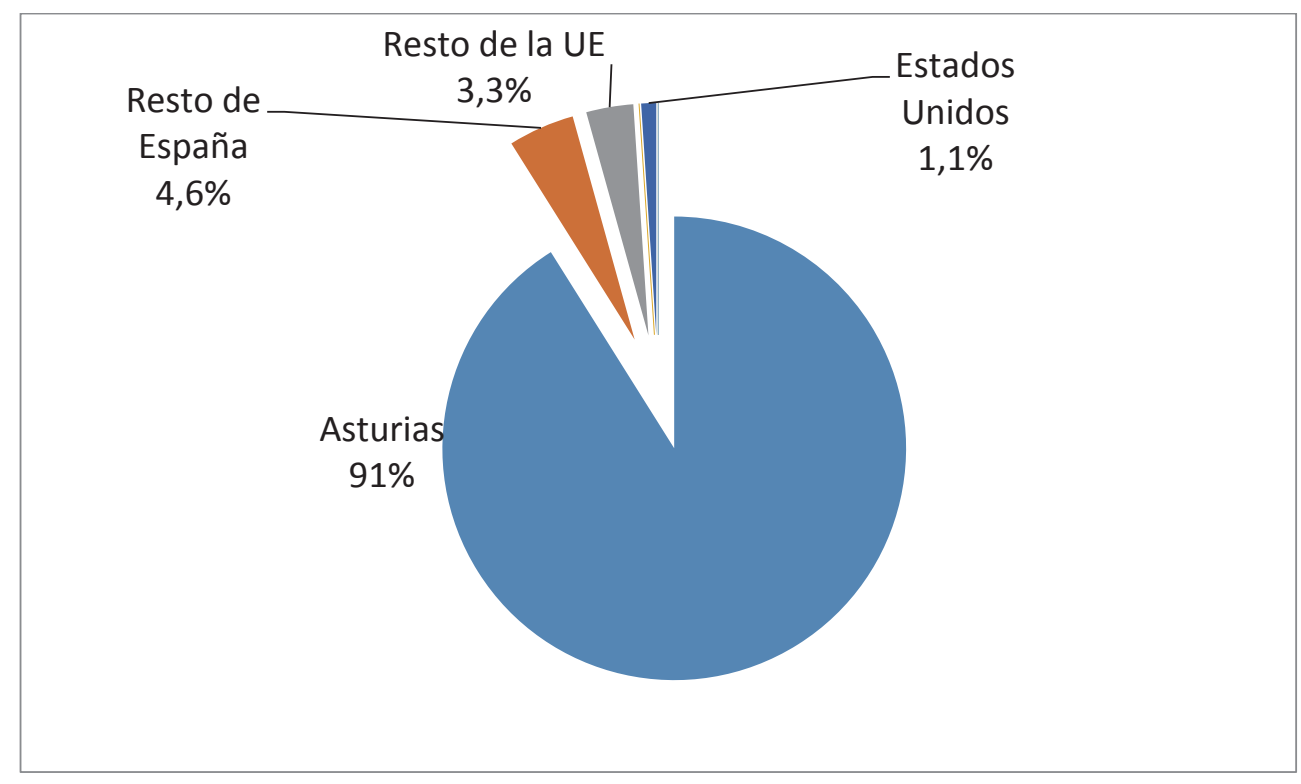

Fuente: Elaboración propia.

Mientras que en Asturias se produce la Información, los contenidos que se importan son del macrogénero Ficción. Como señala Antonio Virgili (entrevista personal, 2018), "acometer un proyecto de ficción [desde Asturias] es un lujo. No se puede. Se han hecho incursiones [pero producir] ficción diaria o semanal, con la situación presupuestaria de la televisión, es inviable". Así, Estados Unidos es el principal expor- 
tador de contenidos de este macrogénero (68,1\% del total), seguido por los originados en el resto de la Unión Europea $(9,8 \%)$ y los de origen mixto $(9,2 \%)$. Estos últimos, se refieren a coproducciones internacionales de telenovelas de Estados Unidos-Venezuela y Estados Unidos-México; así como a coproducciones EspañaArgentina y Australia-Reino Unido.

Por su parte, la ficción representa el 62,1\% del tiempo de emisión de origen comunitario. Se identifica la preeminencia de Reino Unido y Francia, por este orden, como países productores de largometrajes cinematográficos, algunos de ellos en coproducción. Además, destaca la estabilidad en la emisión de la serie "Rex, un policía diferente", una coproducción Austria-Italia que se emite durante varios años.

Gráfico 4. A7-TPA: Contenidos de ficción emitidos según origen, 2006-2014.

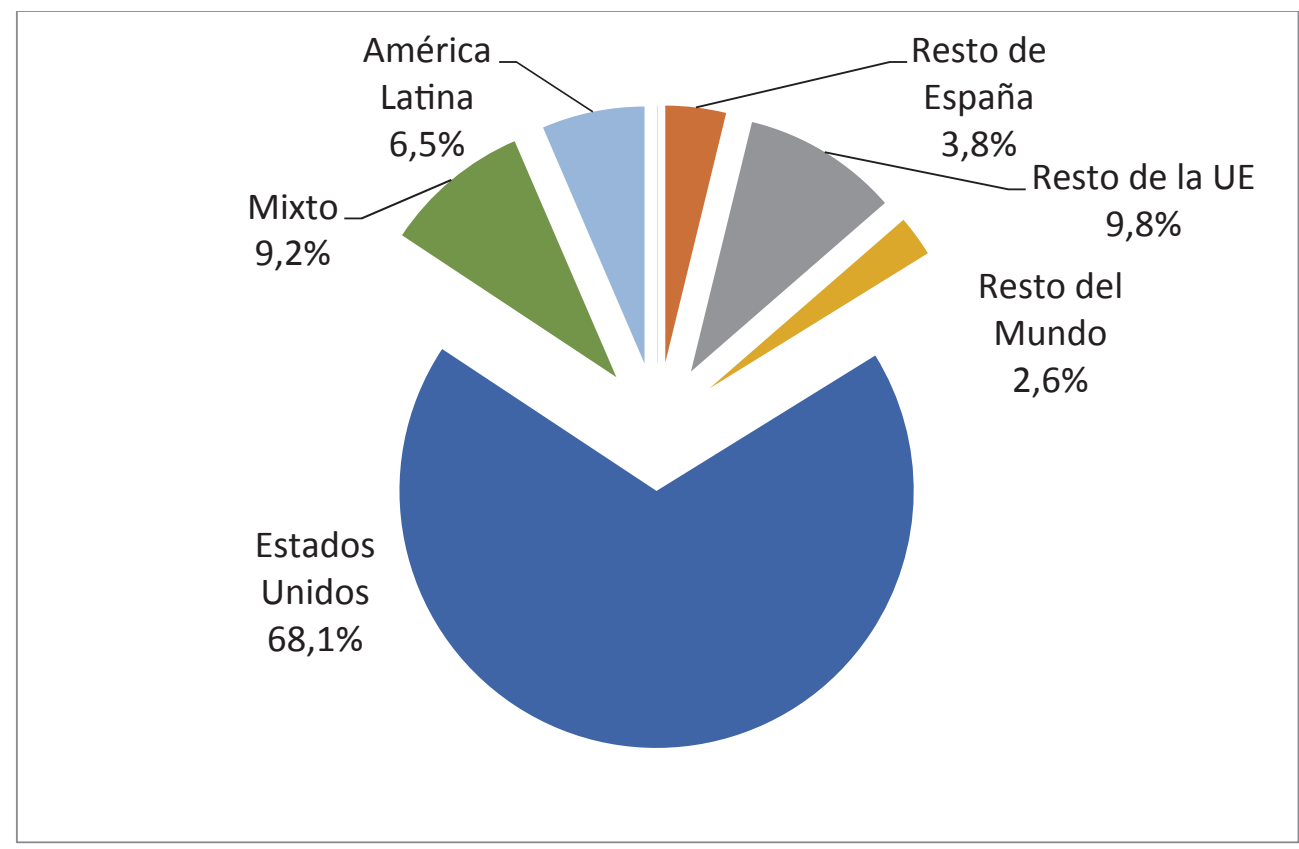

Fuente: Elaboración propia.

Desde el resto de la Unión Europea también se importan programas del macrogénero Información (6,3\%), todos provenientes de Reino Unido (documentales de la televisión pública británica, la British Broadcasting Corporation-BBC-); y del macrogénero Deportes (31,6\%). Estos últimos son, en su mayoría, retransmisiones de la competición europea de fútbol Champions League, que durante el período 20102014 se emitió simultáneamente en las televisiones adscritas a la FORTA con altos niveles de audiencia. Esta apuesta por contenidos premium se concibió como una estrategia de diferenciación ya que su emisión permitía "posicionarnos en el mercado de la Televisión Digital Terrestre con cierta fortaleza" (Pérez Ornia, entrevista personal, 2018). 


\subsection{Diversidad en la lengua de emisión}

La inclusión de la diversidad lingüística en la emisión de $A 7$ se inicia en el año 2007, cuando comienza la emisión de contenidos en asturiano. El castellano y el asturiano son las dos únicas lenguas presentes a lo largo del período analizado. Mientras que la preeminencia del castellano es absoluta durante todo el período, con una media de presencia del $94,4 \%$ del tiempo total de emisión; el asturiano apenas representa el 5,6\%. Aunque este dato es mínimo, es importante destacar su valor simbólico ya que, como se ha señalado, no existe ninguna norma que obligue a las señales de TPA a emitir en la lengua propia del territorio. Como señala Pérez Ornia,

Hay un problema que no está resuelto que es esa dialéctica de si el asturiano tiene o no que ser idioma cooficial y, en ese sentido, no se puede trasladar a TPA la responsabilidad de responder a ese conflicto. Nosotros, creo que de motu propio, hicimos más de lo que teníamos que hacer. [Entre 2007-2011] se dedicó un volumen de dinero que rondaba los tres millones de euros, que en ocasiones llegó a significar más del $20 \%$ de la producción propia. (Pérez Ornia, entrevista personal, 2018)

A partir de 2011, coincidiendo con el cambio en la Dirección General del EPCPA, se incrementa la emisión en asturiano. Un crecimiento que se mantiene hasta el final del análisis y alcanza su nivel máximo en 2013 (11\% del tiempo total de emisión). Este dato constata el interés especial de la dirección de Antonio Virgili por la emisión de programas en lengua asturiana. La importancia de programar estos contenidos responde al cumplimiento de dos objetivos específicos del servicio público de proximidad: la promoción y protección de la lengua propia del territorio, y el impulso al sector audiovisual regional. En este sentido, se certifica que todos los contenidos que se emiten en asturiano se producen en Asturias.

En referencia a su posicionamiento horario en la parrilla de programación de $A 7$, los programas en lengua asturiana se ubican mayoritariamente en la franja Mañana (53\%). Esta franja es la preferida por los programadores de TPA para ubicar los contenidos en lengua asturiana en el período 2007-2011. Sin embargo, a medida que aumenta el tiempo de emisión en asturiano, su concentración en la Mañana disminuye para posicionarse en otras franjas horarias, hasta estar presente en todas ellas en 2014.

Cobra especial relevancia la alta presencia de contenidos en lengua propia en la franja de Prime-Time donde la emisión en asturiano es estable desde 2009, con una media de presencia del $17 \%$. Además, su presencia en esta franja, la de mayor valor para los anunciantes, aumenta a partir de 2012, bajo la dirección de Virgili. Una prueba más del soporte a la emisión de estos contenidos. Este fenómeno también se produce en la franja Mediodía, en tercer lugar, con un 12\% del tiempo de emisión en asturiano; seguida de la Sobremesa con un $10 \%$. Por su parte, las franjas horarias Noche (6\%) y Tarde (2\%) son en las que se localiza la menor presencia de programas en lengua propia. En ambos casos, estos programas se presentan de manera residual y puntual. 
Gráfico 5. A7-TPA: Franja horaria de emisión de los programas en lengua asturiana, 2006-2014.

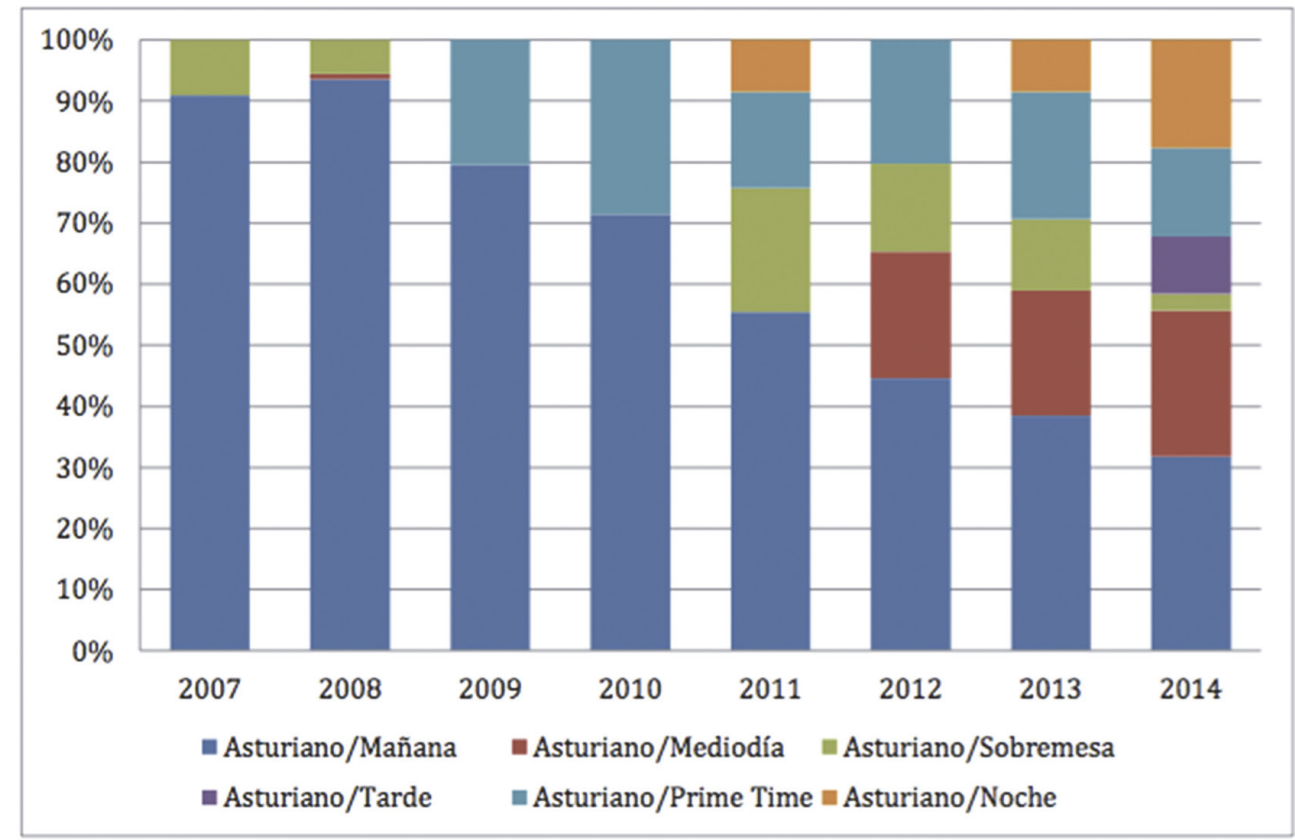

Fuente: Elaboración propia.

Gráfico 6. A7-TPA: Programas emitidos en lengua asturiana según macrogénero, 2006-2014.

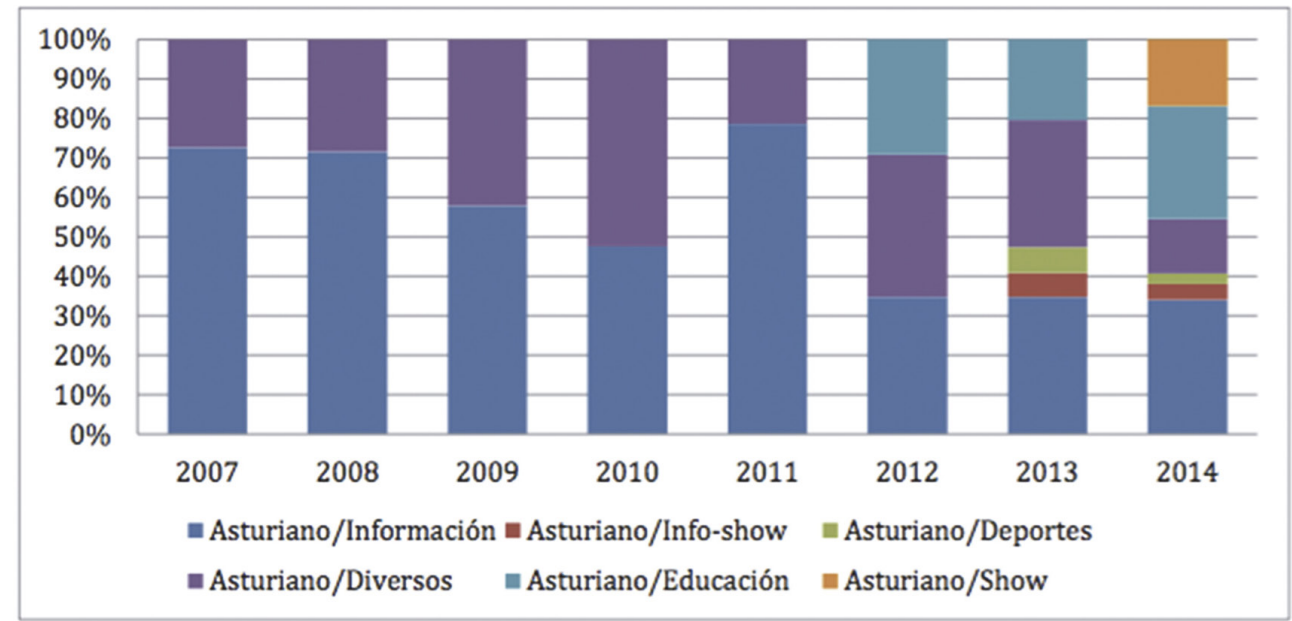

Fuente: Elaboración propia. 
Respecto a los macrogéneros en los que se emite en asturiano, al igual que ocurría con las franjas horarias, se aprecia una diversificación de los mismos a partir de 2012. Mientras que en el período 2007-2011 solo se identifican programas de los macrogéneros Información (66\%) y Diversos (34\%), a partir de 2012 se emiten contenidos de Educación, Deportes, Info-Show y Show. La apuesta por los programas educativos en asturiano, que representan el 14,5\% del tiempo de emisión en asturiano en el trienio 2012-2014, pone otra vez de manifiesto la apuesta de la dirección de Virgili por vincular los objetivos del servicio público de televisión a la normalización lingüística.

\subsection{Diversidad en los macrogéneros}

Si se tiene en cuenta la diversidad genérica de la programación de $A 7$, se identifican nueve de los once macrogéneros propuestos en la tipología de análisis, lo que da cuenta de un efectivo cumplimiento del servicio público en lo que respecta a una oferta programática diversa. Aunque es negativa la ausencia de programas de los macrogéneros Juvenil y Religión, cuya presencia permitiría atender a audiencias específicas habitualmente desatendidas en las televisiones generalistas.

Gráfico 7. A7-TPA: Programas emitidos según macrogénero, 2006-2014.

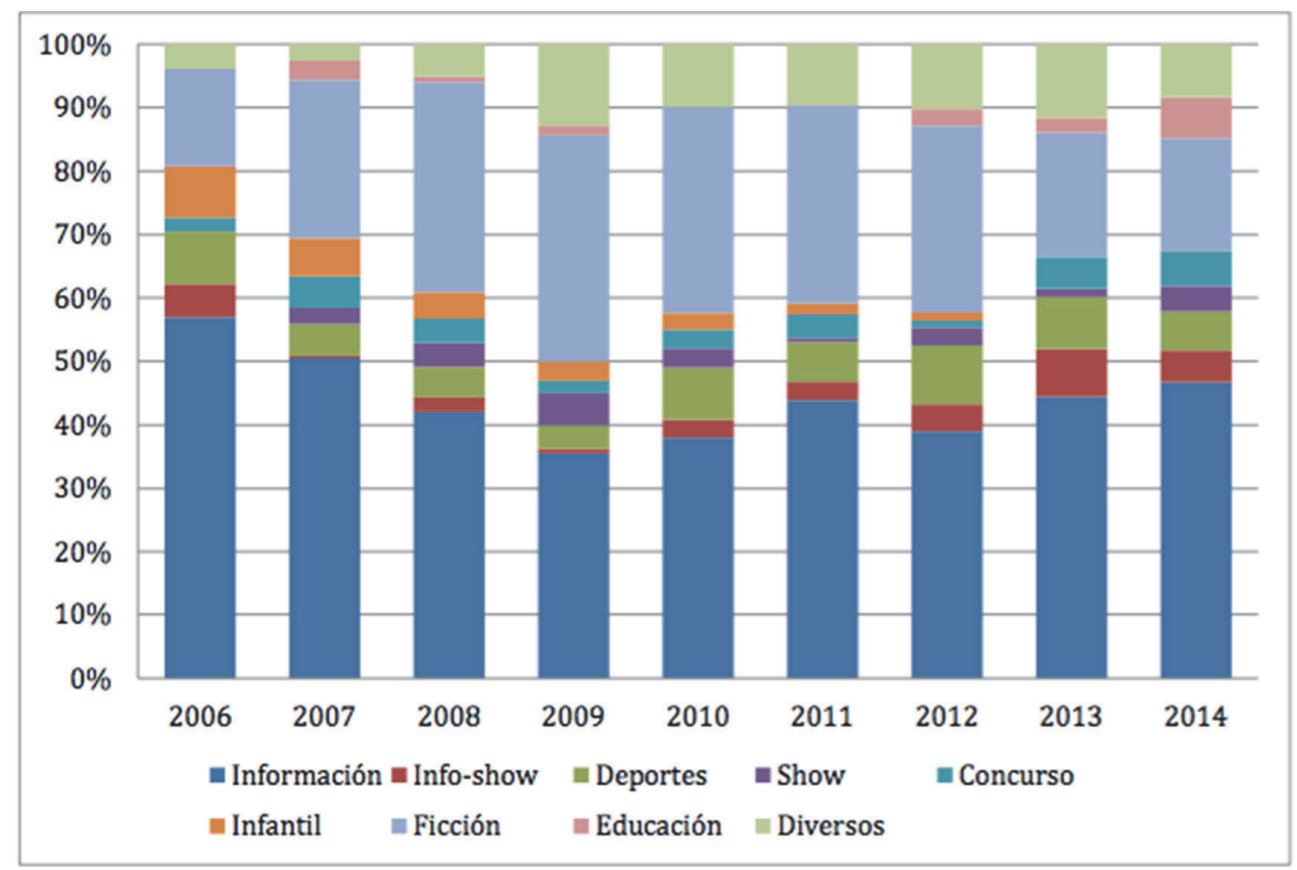

Fuente: Elaboración propia.

La preeminencia de los macrogéneros Información y Ficción es constante durante todo el período 2006-2014, con una media del tiempo de emisión del 44,1\% y del $26,6 \%$, respectivamente. Se confirma que $A 7$ es una señal informativa y que, como 
ocurre en las programaciones de otras televisiones públicas generalistas, la Ficción es clave en su programación. La relación de comportamiento entre ambos macrogéneros muestra que cuando disminuye la importancia de la Información, aumenta la de la Ficción. Así, durante 2009, año de menor presencia porcentual del macrogénero Información $(35,5 \%)$, la Ficción alcanza su máximo dato $(35,7 \%)$ y se sitúa, por única vez, en posición líder en tiempo de emisión.

El mayor volumen de presencia en la emisión del macrogénero Información se corresponde con el primer año de emisión de $A 7$, que es además en el que se localizan el menor número de macrogéneros ofertados, siete. Esto indica que, a pesar del interés de la dirección de TPA por una televisión informativa, las aspiraciones de programación del macrogénero durante el primer año fueron altas. La evolución de presencia del género Información disminuye en el tiempo, al igual que lo hace en otras televisiones públicas, para dar cabida al macrogénero Info-Show (Prado y Delgado, 2010), que se estabiliza en la parrilla de emisión de $A 7$ a partir de 2010 y aumenta gradualmente su presencia hasta el final del período analizado.

El macrogénero Diversos, presente a lo largo de todo el período, es el tercero con mayor representatividad $(8,2 \%)$. Su presencia es discreta durante el trienio 20062008 pero se asienta e intensifica a partir de 2009, con la apuesta continuada por la emisión de programas de folklore y cocina. Por su parte, el macrogénero Deportes se sitúa en cuarto lugar $(6,6 \%$ del total $) \mathrm{y}$, aunque las retransmisiones deportivas son los programas más vistos por la audiencia de $A 7$ (Cañedo, 2018: 244), este macrogénero no supera ningún año el $9 \%$ del total del tiempo de emisión.

En cuanto al resto de macrogéneros, su presencia es mínima y no superan la barrera del 3,5\% del tiempo de emisión. Así, aunque la presencia de los macrogéneros Concurso (3,5\%), Info-Show (3,5\%), Infantil (3\%), Show (2,6\%) y Educación (1,9\%) aportan variedad a la diversidad genérica de la parrilla $A 7$; en términos de equilibrio, su presencia es minoritaria e inestable.

Se considera especialmente negativo para el cumplimiento del servicio público la escasa presencia, en tiempo de emisión, de los contenidos Infantil y Educación y la ya señalada ausencia de contenidos del macrogénero Juvenil. Esta investigación ha constatado que, aunque existe la voluntad de programar contenidos específicos destinados a audiencias infantiles y juveniles, el bajo presupuesto asignado a TPA impide "atender a todas las demandas sociales" (Virgili, entrevista personal, 2018).

\section{Conclusiones y propuestas}

El análisis realizado a la parrilla de programación de $A 7$, en el período comprendido entre el inicio de las emisiones regulares de TPA y su reconversión empresarial en 2015, permite definir a la televisión autonómica asturiana como una televisión informativa, de origen asturiano y que emite en castellano.

Respecto a la diversidad aportada por los contenidos emitidos en $A 7$, se concluye que:

- TPA es una televisión asturiana e informativa de proximidad, de acuerdo a lo reflejado en su normativa y a sus responsabilidades de servicio público. La mayor parte de los contenidos son producidos por empresas asturianas lo que permite cumplir con el compromiso asumido de dinamizar el sector audiovi- 
sual regional. Estas empresas asturianas, además, producen la mayor parte de la información que se emite.

- TPA emite una programación generalista similar a la de otras televisiones públicas. La información de proximidad y la ficción estadounidense ocupan la mayor parte del tiempo de emisión en $A 7$. Estados Unidos es el segundo productor de contenidos mientras que la presencia de programas de otros orígenes es minoritaria. En este sentido, no se cumple con lo establecido en los principios y criterios de programación del EPCPA que señalaban el especial interés en programar contenidos comunitarios y latinoamericanos.

- TPA incumple los principios y criterios de programación respecto a la promoción de la diversidad lingüística ya que la presencia del asturiano, la lengua propia del territorio, supone poco más del 5\% del total del tiempo de emisión. $A 7$ es, por tanto, una señal de televisión que emite en castellano. No obstante, se considera positiva esta escasa presencia ante la inexistencia de norma alguna que obligue a la programación de contenidos en lengua asturiana. En este sentido, se ha constatado que la presencia de contenidos en asturiano en la parrilla de emisión de $A 7$ está directamente vinculada con la voluntad de la dirección general de la televisión autonómica. Además, se certifica que toda la producción en lengua asturiana es realizada por empresas asturianas, lo que permite, así mismo, contribuir al cumplimiento del objetivo de dinamizar el sector audiovisual regional.

- TPA programa una amplia variedad de macrogéneros en términos de desequilibrio dada la preeminencia de la Información y la Ficción. Se destaca negativamente la mínima presencia de contenidos infantiles y educativos y la ausencia de contenidos juveniles y religiosos. Esta desatención a la totalidad de las demandas de la población, aunque es habitual en las programaciones generalistas de las televisiones públicas, dificulta el efectivo cumplimiento de los objetivos de servicio público encomendados. Dicha carencia se justifica, principalmente, en la escasez presupuestaria de la televisión autonómica.

A fin de garantizar una mayor efectividad en el cumplimiento del servicio público de proximidad encomendado a TPA, se propone:

- La aprobación, con carácter de urgencia, del mandato marco regulado en la normativa de la televisión autonómica. El desarrollo, a posteriori, de diversos contratos programa que implementen dicho mandato permitirá garantizar los objetivos de servicio público marcados. Respecto a la diversidad ofertada, en términos de origen geográfico de los contenidos, lengua de emisión y macrogéneros se recomienda el establecimiento de cuotas de obligado cumplimiento.

- El desarrollo de una política lingüística por parte de la televisión autonómica con respecto a la lengua propia. Dada la actual situación de minorización de la lengua asturiana, se considera que la simple recomendación de su uso es insuficiente. En este sentido, la televisión pública debe liderar la normalización lingüística en la región, como ha ocurrido en otras regiones con televisión autonómica.

- El incremento de los presupuestos de actividad de la televisión autonómica de tal manera que se garantice el desarrollo de programaciones que atiendan 
a sectores minoritarios de la sociedad. Se considera como prioridad la programación de contenidos infantiles y juveniles que permitan desarrollar el rol educativo del servicio público.

\section{Referencias bibliográficas}

Albornoz, L. A. (Comp.) (2011). Poder, Medios, Cultura. Una mirada critica desde la Economía Política de la Comunicación. Buenos Aires: Paidós.

Albornoz, L. A. y García Leiva, M. T. (Eds.) (2017a). El audiovisual en la era digital. Políticas y estrategias para la diversidad. Madrid: Cátedra.

- (Eds.) (2017b). Diversidad e industria audiovisual: el desafio cultural del siglo XXI. Ciudad de México: Fondo de Cultura Económica.

Bustamante, E. y Zallo, R. (Coords.) (1988). Las industrias culturales en España (grupos multimedia y transnacionales). Madrid: Akal.

Campos Freire, F. (Coord.) (2016). Situación actual y tendencias de la radiotelevisión pública en Europa. Santiago de Compostela: Universidad de Santiago de Compostela / FORTA.

Cañedo, A. (2018). “Televisión del Principado de Asturias: dinamización y diversidad en el sector audiovisual asturiano (2005-2015)". Tesis doctoral. Universidad Carlos III de Madrid.

Delgado, M.; Prado, E.; y Navarro, C. (2017). Ficción televisiva en Europa (EU5): origen, circulación de productos y puesta en parrilla. El profesional de la información (26) 1 , 132-140.

Ente Público de Comunicación del Principado de Asturias (EPCPA). (2006). Principios y criterios de programación de los medios del Ente Publico de Comunicación del Principado de Asturias: texto aprobado por el Consejo de Administración en su reunión de fecha 23 de enero de 2006 e informado por el Consejo de Comunicación. Oviedo: EPCPA.

Frau-Meigs, D. (2002). Excepción cultural, políticas nacionales y mundialización. Factores de democratización y de promoción de lo contemporáneo. Quaderns del CAC, 14, 3- 18.

Gutiérrez, E. (Coord.) (2017). Televisión Abierta. Situación actual y Tendencias de futuro de la TDT. Madrid: Colegio Oficial de Ingenieros de Telecomunicación.

Hevia, I. (2018). Los jóvenes y la lengua asturiana. Oviedo: Conseyu de la Mocedá del Principáu d'Asturies / Academia de la Llingua Asturiana.

Marzal, J. (Ed.) (2015). Las televisiones públicas autonómicas del siglo XXI. Nuevos escenarios tras el cierre de RTVV. Bellaterra: Universidad Autónoma de Barcelona.

Marzal, J.; Izquierdo, J. y Casero, A. (Eds.) (2015). La crisis de la televisión pública. El caso de RTVV y los retos de una nueva gobernanza. Bellaterra: Universidad Autónoma de Barcelona.

Mattelart, A. (2006). Diversidad cultural y mundialización. Barcelona: Paidós.

Mazzioti, N. (1996). La industria de la telenovela: la producción de Ficción en América Latina. Barcelona: Paidós.

McQuail, D. (1998). La acción de los medios. Los medios de comunicación y el interés público. Buenos Aires: Amorrortu.

Moragas, M.; Garitaonandía, C. y López, B. (Eds.) (1999). Televisión de proximidad en Europa. Experiencias de descentralización en la era digital. Bellaterra: Universidad Autónoma de Barcelona. 
Murdock, G. (1994). New times/hard times: Leisure, participation and the common good. Leisure Studies, 13, 239-248.

Napoli, P. (1999). Deconstructing the Diversity Principle. Journal of Communication, (49) 4, 7-34.

Pasquali, A. (1991). El orden reina. Escritos sobre comunicación. Caracas: Monte Ávila.

Prado, E. (Dir.) (2017). Diversidad e industrias culturales. Quaderns del CAC (XX) 43.

Prado, E. y Delgado, M. (2010). La televisión generalista en la era digital. Tendencias internacionales de programación. Telos, 84, 52-64.

Ranaivoson, H. (2005). The economic analysis of product diversity. Cahiers de la MSE. París: Centre national de la recherche scientifique.

- (2007). Measuring cultural diversity: a review of existing definitions. París: UNESCO-UIS.

Richeri, G. (1983). La televisión entre servicio público y negocio. Barcelona: Gustavo Gili.

Stirling, A. (1998). On the economics and analysis of diversity. SPRU Electronic Working Paper, 28.

- (2007). A general framework for analysing diversity, in science, technology and society. Journal of the Royal Society Interface, 4, 707-719.

UNESCO. (2006). Radiotelevisión de servicio público: un manual de mejores prácticas. San José: UNESCO.

UNESCO-UIS. (2011). Measuring the diversity of cultural expressions: applying the Stirling model of diversity in culture. Two case studies (Technical Paper No. 6). Montreal: UNESCO-UIS.

Van der Wurff, R. (2004). Supplying and Viewing diversity: The Role of Competition and Viewer Choice in Dutch broadcasting. European Journal of Communication, (19) 2, 215 237.

- (2005). Competition, Concentration and Diversity in European Television. Journal of Cultural Economics, 29, 249-275.

Zallo, R. (2011). Estructuras de la comunicación y de la cultura. Políticas para la era digital. Barcelona: Gedisa. 